\title{
Tratamiento con soportes plantares en la enfermedad de Müller-Weiss. Reporte preliminar
}

\author{
Treatment with plantar supports in Müller-Weiss disease. Preliminary report
}

\author{
Ruiz-Escobar J,*,‡Viladot-Pericé R,* Álvarez-Goenaga F, ${ }^{*, \S}$ Ruiz-Escobar P," Rodríguez-Boronat E* \\ Clínica Tres Torres-Barcelona, España.
}

RESUMEN. El objetivo de este trabajo es valorar la eficacia del tratamiento conservador con plantillas ortopédicas en la enfermedad de Müller-Weiss (EMW). El soporte plantar tiene como objetivo pronar el retropié e inmovilizar las articulaciones talonavicular y naviculocuneiforme o ambas a la vez como alternativa a la osteotomía valguizante de calcáneo y las artrodesis. Se analizan las características clínicas y radiológicas de 10 casos de EMW en ocho pacientes objeto del estudio y se valoran los resultados obtenidos. Se comenta el proceso de fabricación de los soportes plantares así como los materiales empleados. El tamaño de la muestra no es significativo; sin embargo, de manera preliminar, el tratamiento conservador es posible en determinados casos de la EMW para aliviar el dolor, mejorar la dinámica de la marcha y limitar la progresión de la enfermedad en el corto plazo.

Palabras clave: Enfermedad de Müller-Weiss, tratamiento conservador.

\section{Nivel de evidencia: IV}

* Clínica Tres Torres-Barcelona.

‡ Pie y Salud Centro de Especialidades. La Vall d’Uixó (Castellón).

${ }^{\S}$ Hospital San Rafael. Barcelona.

" Pieytobillo. Clínica Quirón-Rotger. Palma de Mallorca.

España.

Dirección para correspondencia: Javier Ruiz-Escobar

C/Salvador Allende, 4-2 C. 12600 , La Vall d’Uixó (Castellón), España, Tel. +34-625803973

E-mail: javierpodologia@pieysalud.es

https://dx.doi.org/10.35366/95325

doi: $10.35366 / 95325$
ABSTRACT. The objective of this work is to assess the efficacy of conservative treatment with orthopedic insoles in Müller-Weiss disease (EMW). The plantar support aims to pronounce the hindfoot and immobilize the talo-navicular, and naviculo-cuneiform joints or both at the same time, as an alternative to the valguizing osteotomy of the calcaneus and arthrodesis. The clinical and radiological characteristics of 10 cases of EMW in 8 patients under study are analyzed and the results obtained are evaluated. The manufacturing process of the plantar supports is commented, as well as the materials used. The sample size is not significant, however, preliminary, conservative treatment is possible in certain cases of EMW to relieve pain, improve gait dynamics and limit disease progression in the short-term.

Keywords: Müller-Weiss disease, conservative treatment.

\section{Introducción}

La enfermedad de Müller-Weiss (EMW) es conocida desde el año 1927, en el que Walter Müller, ${ }^{1}$ cirujano ortopédico de la ciudad alemana de Leipzig describió el primer caso y Konrad Weiss, ${ }^{2}$ radiólogo austriaco, dos nuevos casos.

La etiología de la EMW es motivo de controversia. Se ha relacionado con una necrosis ósea, hipótesis defendida por varios autores. ${ }^{3,4,5,6,7,8}$ Influyó en ello que Weiss ${ }^{2}$ trabajara con Robert Kiemböck, que había descrito la necrosis del semilunar. Sin embargo, Rochera ${ }^{9}$ en 1981 publicó un trabajo sobre 46 pies afectos de EMW, realizándose en nueve de ellos una biopsia ósea. En ningún caso hubo hallazgos compatibles con una necrosis. Monteagudo y Maceira ${ }^{10}$ tampoco han encontrado signos de necrosis ósea en sus casos.

La EMW también se ha relacionado con otras causas ${ }^{11}$ como un retraso en la aparición del núcleo de osificación, 

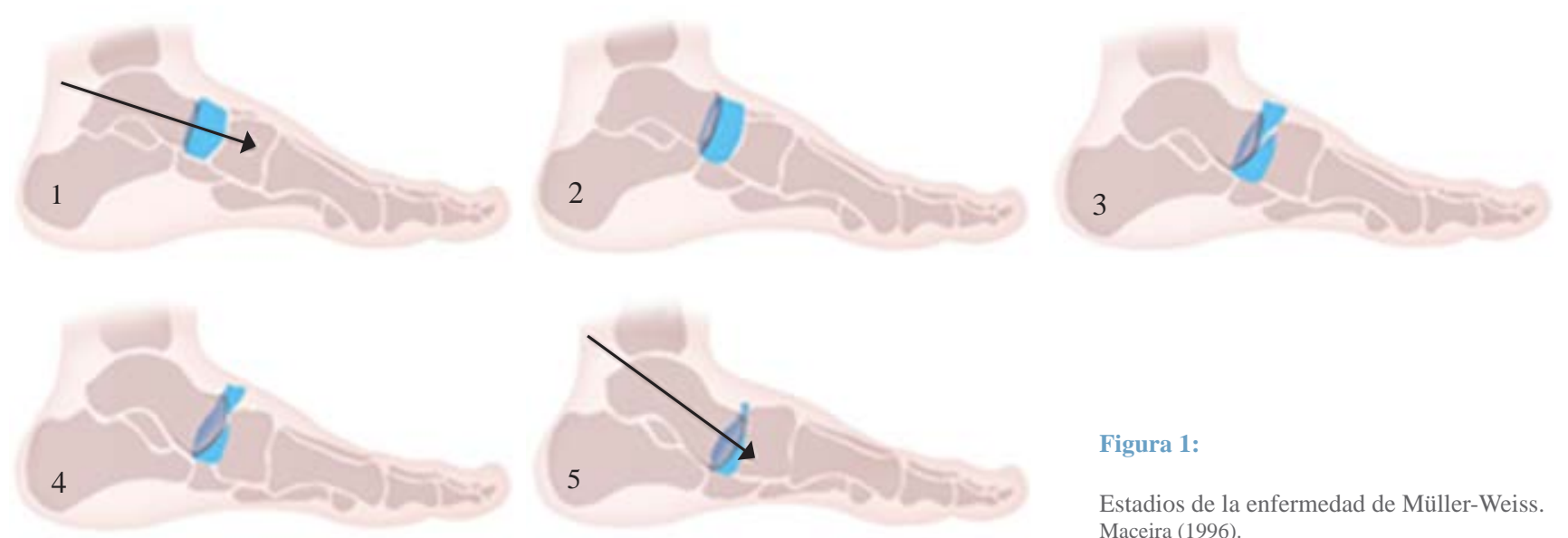

Figura 1: Maceira (1996).
Estadios de la enfermedad de Müller-Weiss. un navicular bipartito, una osteocondritis de origen postraumático como secuela de una enfermedad de Köhler I, en relación con una coalición tarsiana calcáneo-navicular o bien otro tipo de malformación congénita, etc., entre otras posibilidades que pueden afectar la estructura ósea del navicular durante el crecimiento.

Desde un punto de vista biomecánico hay un aumento de cargas en el tercio lateral del navicular en sentido posteroanterior, favorecida por una menor divergencia talocalcánea. En el tercer rocker de la marcha, la presión en el tercio lateral del navicular procede del segundo metatarsiano y el segundo cuneiforme, ya que por lo general estos pacientes tienen una fórmula metatarsiana tipo index minus.

Así pues, sobre un hueso cuya estructura no es normal, se producen unas cargas que alteran su morfología, por todo ello en la actualidad se considera a EMW como una displasia del navicular. ${ }^{10} \mathrm{El}$ origen de la lesión se produce durante la infancia y se hace sintomática en la edad adulta. En los deportistas, la existencia de una mayor solicitación biomecánica, con cambios de velocidad y dirección súbitas y constantes, la clínica puede manifestarte en edades más tempranas.

En muchos casos el pie presenta el aspecto de un «pie plano paradójico» ${ }^{12}$ ya que el talón está en varo. Por lo general el dolor aparece a partir de los 40 años, derivado de la afectación que de manera progresiva se instaura a nivel de las articulaciones talo-navicular y naviculocuneiforme. Los pacientes refieren dolor en la parte dorsal y medial del pie y en ocasiones en el seno del tarso. La bipedestación y las marchas prolongadas propician la aparición y progreso de la sintomatología.

Maceira ${ }^{13}$ en 1996 clasificó en cinco estadios las alteraciones radiológicas en el plano sagital de la enfermedad (Figura 1). En el estadio III ya puede observarse la fractura por insuficiencia del navicular que, como publicó Rochera, ${ }^{9}$ tiene un trazo de $45^{\circ}$ en la que hay un fragmento dorsal y medial y otro plantar y lateral que está en relación con el defecto estructural y morfológico del navicular, que se encuentra sometido a cargas norma- les (Figura 2). No debe confundirse con una fractura por estrés, que se produce por una sobrecarga sobre un hueso normal.

Con referencia al tratamiento conservador, hace unos años en ocasiones se indicaban plantillas de inmovilización, sin valorar la compensación del varo de retropié.

La artrodesis talonavicular en el retropié ha sido la técnica más utilizada. ${ }^{14,15,16,17}$ Nosotros realizábamos una artrodesis talonaviculocuneiforme mediante un injerto en marquetería procedente de la tibia. Los resultados eran discretos y las molestias persistían en muchas ocasiones. Por lo general no se realizaba ningún gesto de corrección del varo de talón.

Actualmente ha cambiado este enfoque dando absoluta prioridad a la corrección del varo de talón mediante una osteotomía valguizante de calcáneo. ${ }^{18}$ En casos de severa artrosis de las articulaciones mediales se puede asociar

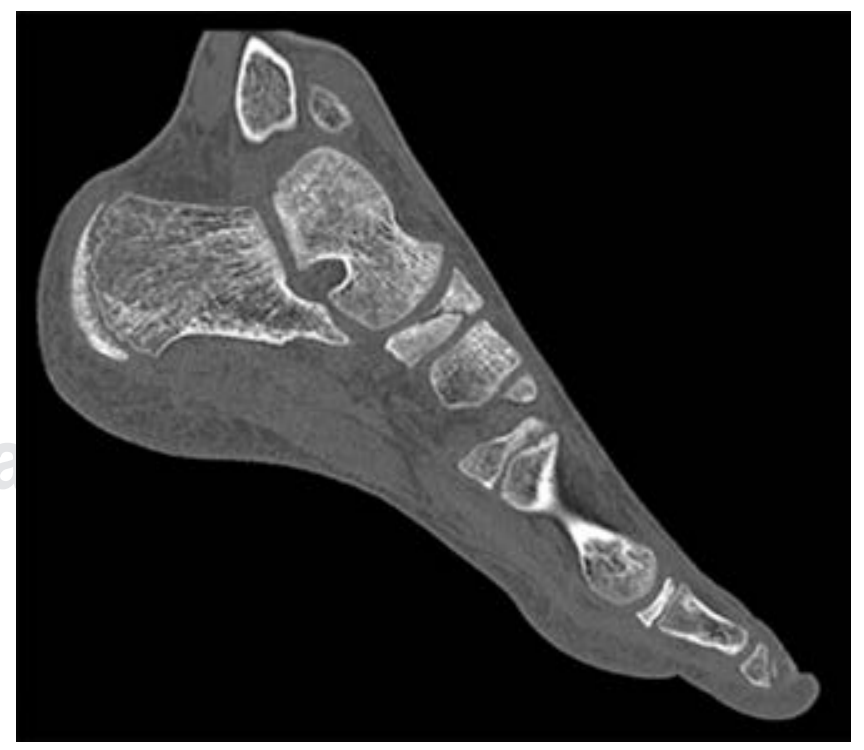

Figura 2: Resonancia magnética de plano sagital. Fractura por insuficiencia del navicular con un trazo de $45^{\circ}$ en el estadio III de la enfermedad de Müller-Weiss. 
una artrodesis a dicho nivel (Figuras 3 A-D). El objetivo de este trabajo es valorar si con un soporte plantar a medida, podemos obtener los mismos resultados que con la cirugía: corregir o compensar la desviación en varo del retropié y además inmovilizar el arco interno. Las preguntas que nos planteamos son: ¿cuándo estarían indicados los soportes plantares a medida?, ¿cómo debe realizarse la plantilla?, ¿cuáles son los resultados?
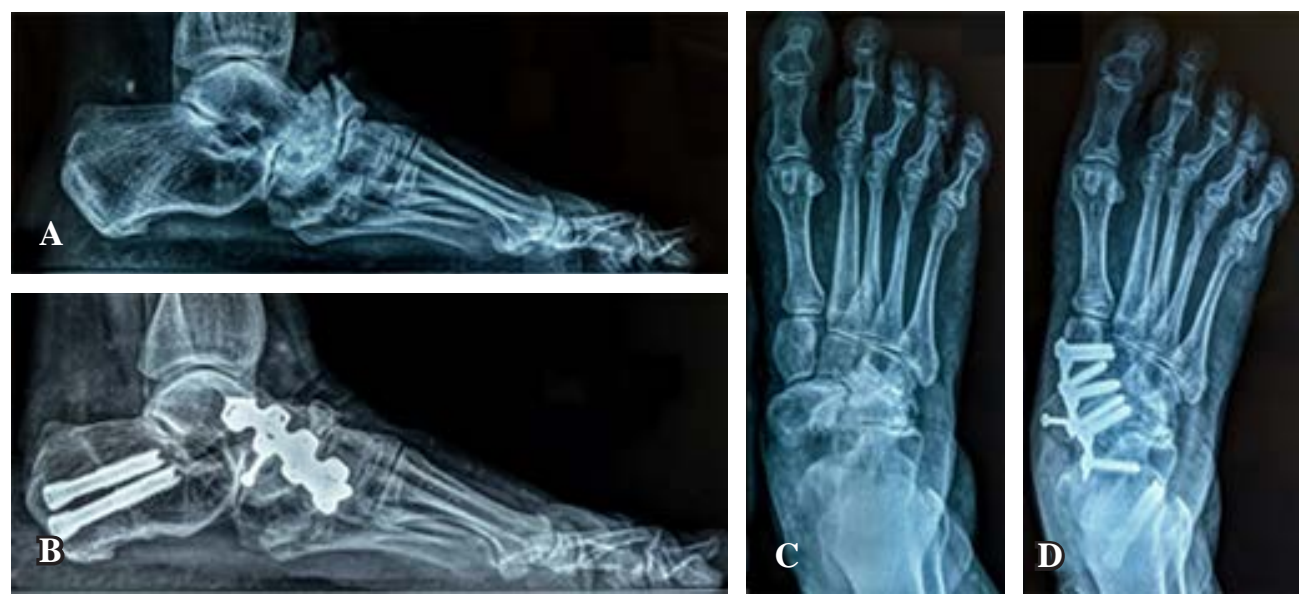

Figura 3:

Caso 4, estadio V. Osteotomía de calcáneo y artrodesis talonaviculocuneiforme. A y C) Preoperatorio; B y D) Postoperatorio.

Tabla 1: Estadios de la enfermedad de Müller-Weiss.

\begin{tabular}{|c|c|c|c|c|c|c|c|c|c|}
\hline Casos & Sexo & Edad & $\begin{array}{l}\text { Primera } \\
\text { visita }\end{array}$ & Seguimiento & $\begin{array}{c}\text { Localización } \\
\text { dolor }\end{array}$ & $\begin{array}{l}\text { Estadio } \\
\text { radioló- } \\
\text { gico }\end{array}$ & $\begin{array}{l}\text { Actividad/ } \\
\text { deporte }\end{array}$ & $\begin{array}{l}\text { Tratamiento } \\
\text { soporte plantar }\end{array}$ & $\begin{array}{l}\text { Tratamiento } \\
\text { cirugía }\end{array}$ \\
\hline 1 & Femenino & 57 & $\begin{array}{l}\text { Marzo } \\
2012\end{array}$ & $\begin{array}{l}\text { Siete años y } \\
\text { ocho meses }\end{array}$ & $\begin{array}{l}\text { Seno tarso y } \\
\text { subastraga- } \\
\text { lina PI }\end{array}$ & II & No & $\begin{array}{l}\text { Polipropileno } \\
2 \text { mm con cuña } \\
\text { pronadora PI }\end{array}$ & No \\
\hline 2 & Femenino & 46 & $\begin{array}{l}\text { Octubre } \\
2013\end{array}$ & $\begin{array}{l}\text { Seis años y } \\
\text { un mes }\end{array}$ & $\begin{array}{c}\text { Dorso } \\
\text { medial PI }\end{array}$ & II & Sí & $\begin{array}{l}\text { Polipropileno } \\
3 \text { mm con cuña } \\
\text { pronadora PD }\end{array}$ & No \\
\hline 3 & Femenino & 68 & $\begin{array}{l}\text { Febrero } \\
2015\end{array}$ & $\begin{array}{l}\text { Cuatro años } \\
\text { y nueve } \\
\text { meses }\end{array}$ & $\begin{array}{c}\text { Dorso } \\
\text { medial PI }\end{array}$ & IV & No & $\begin{array}{l}\text { Polipropileno } \\
3 \text { mm con cuña } \\
\text { pronadora PI }\end{array}$ & $\begin{array}{c}\text { Cirugía osteotomía } \\
\text { valguizante calcá- } \\
\text { neo + artrodesis } \\
\text { talo navicular }\end{array}$ \\
\hline 4 & Femenino & 68 & $\begin{array}{c}\text { Febrero } \\
2015\end{array}$ & $\begin{array}{l}\text { Cuatro años } \\
\text { y nueve } \\
\text { meses }\end{array}$ & $\begin{array}{c}\text { Dorso } \\
\text { medial PD }\end{array}$ & V & No & $\begin{array}{l}\text { Polipropileno } \\
3 \text { mm con cuña } \\
\text { pronadora PI }\end{array}$ & $\begin{array}{c}\text { Cirugía osteotomía } \\
\text { valguizante calcá- } \\
\text { neo + artrodesis } \\
\text { talo naviculocu- } \\
\text { neiforme }\end{array}$ \\
\hline 5 & Femenino & 59 & $\begin{array}{l}\text { Marzo } \\
2015\end{array}$ & $\begin{array}{l}\text { Cuatro } \\
\text { años y ocho } \\
\text { meses }\end{array}$ & $\begin{array}{c}\text { Dorso } \\
\text { medial PD }\end{array}$ & II & No & $\begin{array}{c}\text { Polipropileno } \\
3 \text { mm con cuña } \\
\text { pronadora PD }\end{array}$ & No \\
\hline 6 & Masculino & 46 & $\begin{array}{l}\text { Julio } \\
2015\end{array}$ & $\begin{array}{l}\text { Cuatro años } \\
\text { y cuatro } \\
\text { meses }\end{array}$ & $\begin{array}{l}\text { Dorso me- } \\
\text { dial y seno } \\
\text { del tarso PD }\end{array}$ & II & No & $\begin{array}{l}\text { Polipropileno } \\
3 \text { mm con cuña } \\
\text { pronadora PD + } \\
\text { infiltración seno } \\
\quad \text { del tarso }\end{array}$ & No \\
\hline 7 & Masculino & 13 & $\begin{array}{l}\text { Noviembre } \\
2015\end{array}$ & Cuatro años & $\begin{array}{c}\text { Dorso } \\
\text { medial PI }\end{array}$ & III & Sí & $\begin{array}{l}\text { Polipropileno } \\
3 \text { mm con cuña } \\
\text { pronadora PI }\end{array}$ & No \\
\hline 8 & Masculino & 38 & $\begin{array}{l}\text { Marzo } \\
2018\end{array}$ & $\begin{array}{c}\text { Un año y } \\
\text { ocho meses }\end{array}$ & $\begin{array}{c}\text { Dorso } \\
\text { medial PI }\end{array}$ & II & Sí & $\begin{array}{l}\text { Polipropileno } \\
4 \text { mm con cuña } \\
\text { pronadora PI }\end{array}$ & No \\
\hline 9 & Masculino & 64 & $\begin{array}{c}\text { Octubre } \\
2018\end{array}$ & $\begin{array}{c}\text { Un año } \\
\text { y un mes }\end{array}$ & $\begin{array}{c}\text { Dorso } \\
\text { medial PI }\end{array}$ & II & No & $\begin{array}{l}\text { Polipropileno } \\
3 \text { mm con cuña } \\
\text { pronadora PI }\end{array}$ & No \\
\hline 10 & Masculino & 64 & $\begin{array}{l}\text { Octubre } \\
2018\end{array}$ & $\begin{array}{c}\text { Un año } \\
\text { y un mes }\end{array}$ & $\begin{array}{c}\text { Dorso } \\
\text { medial PD }\end{array}$ & III & No & $\begin{array}{c}\text { Polipropileno } \\
3 \text { mm con cuña } \\
\text { pronadora PI }\end{array}$ & $\begin{array}{l}\text { Osteotomía } \\
\text { valguizante de } \\
\text { calcáneo }\end{array}$ \\
\hline
\end{tabular}



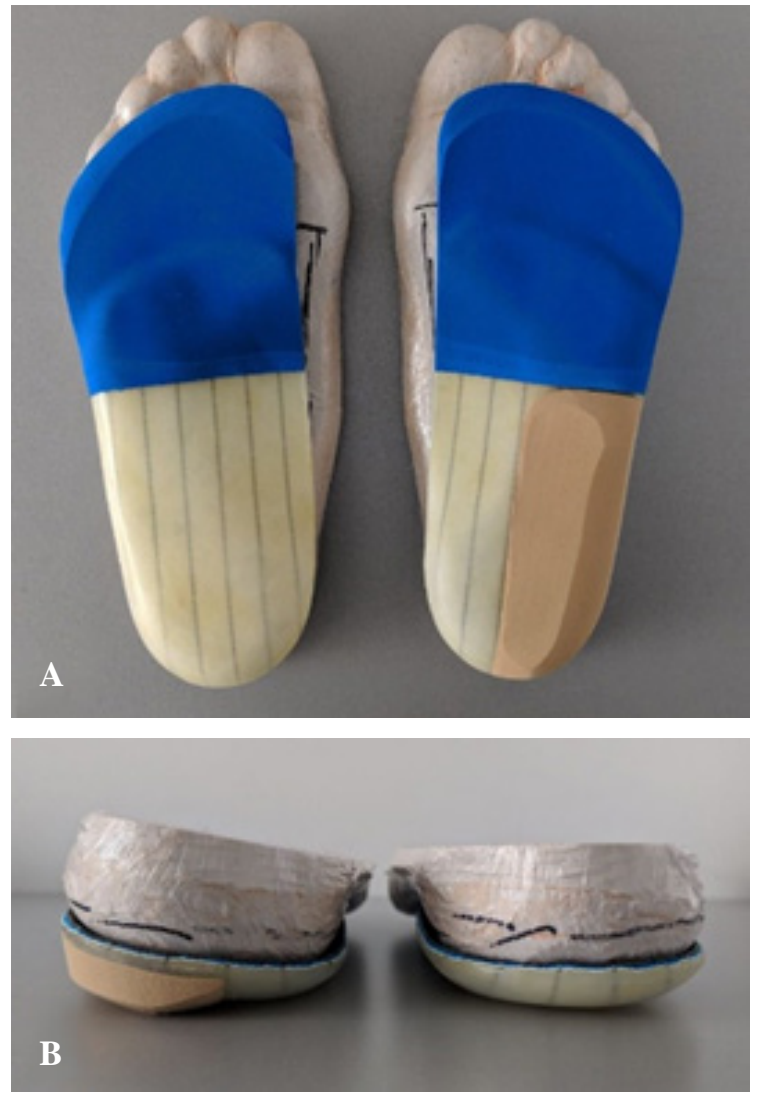

Figura 4: Plantillas finalizadas adaptadas a los moldes de un paciente con enfermedad de Müller-Weiss. A) visión plantar y B) visión posterior.

\section{Material y métodos}

Se estudian 10 casos en ocho pacientes afectos de EMW que fueron tratados con soportes plantares a medida. En todos ellos se analizan las siguientes variables: sexo, edad, lado, seguimiento, localización del dolor, estadio radiológico (Maceira ${ }^{13}$ ) actividades de la vida diaria (deporte), características de las plantillas utilizadas, casos intervenidos y tipo de cirugía (Tabla 1).

Se utilizó en todos los pacientes el mismo modelo de plantilla, cuyo objetivo era corregir o compensar el varo de talón e inmovilizar el arco medial. Es fundamental, para que la ortesis sea efectiva, que la prescripción sea correcta y que el médico y podólogo tengan una buena comunicación.
En la fabricación de la plantilla los materiales empleados han sido, como base del soporte plantar, polipropileno de $3 \mathrm{~mm}$ de grosor, recurriendo en un caso a $4 \mathrm{~mm}$ de grosor debido a la envergadura del paciente, al que se le añade en el pie afecto una cuña pronadora total de un EVA de alta densidad de $6 \mathrm{~mm}$ de grosor pulido a cero de lateral a medial, desde talón hasta la zona retrocapital de las cabezas metatarsianas 4-5 (Figura 4 A y B).

El polipropileno es una lámina termoplástica que calentado a unos $200{ }^{\circ} \mathrm{C}$ se hace maleable permitiendo su adaptación al molde de un pie mediante el prensado que aplica un vacuum o unidad de vacío, el cual utiliza una lámina de vinilo o látex para que la adaptación sea fiel al molde. EVA o etilvinilacetato es un polímero termoplástico ampliamente utilizado en el mundo del calzado, especialmente deportivo para fabricar las suelas, entre otras aplicaciones. Su densidad es muy variable, pudiendo ser muy blandas o de mayor rigidez.

La captura de los moldes supone un elemento esencial en la fabricación de los soportes. De los múltiples sistemas conocidos, recomendamos la captura de la muestra en carga, sin realizar ninguna maniobra de compensación o neutralización. En nuestro caso, optamos por la captura de la huella plantar mediante espumas fenólicas (Figura 5).

Una vez obtenido el positivo de los moldes, es importante a la hora de estabilizarlo no realizar una corrección importante del arco medial. En personas de mediana y avanzada edad, la corrección a este nivel no es tolerada persistiendo o aumentando las molestias existentes. El objetivo es por tanto, mejorar la adaptación del polipropileno para ferulizar sin pretender con ello corregir el aplanamiento del pie.

Con el fin de delimitar el área que comprende el arco del pie, debemos tener en cuenta la zona aproximada donde se localizan las cabezas primera y quinta metatarsianas trazando un arco imaginario que correspondería a la parábola metatarsiana. Acto seguido, trazaremos una bisectriz que se extenderá desde tercer espacio interdigital hasta la mitad aproximada del retropié dejando libre finalmente toda el área de apoyo del talón. Con estas referencias podremos acotar la zona en la cual marcaremos el arco longitudinal medial del pie (Figura 6).

Con el soporte pulido, añadimos finalmente una cuña pronadora total de aproximadamente $6 \mathrm{~mm}$ de grosor en

Figura 5:
Captura de moldes en carga
mediante espuma fenólica.
mediante espuma fenólica.

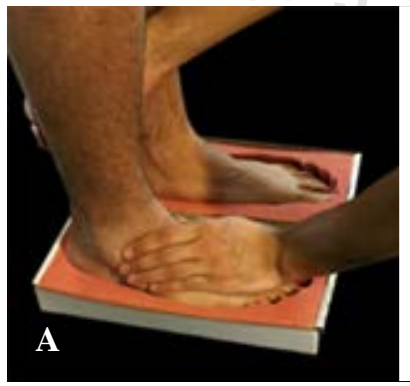

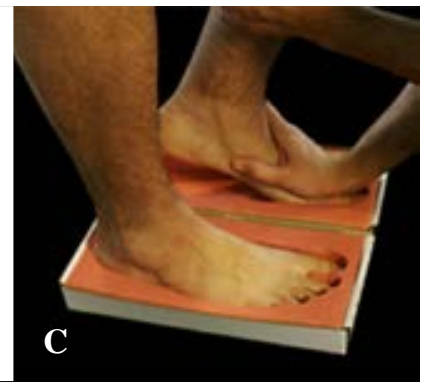




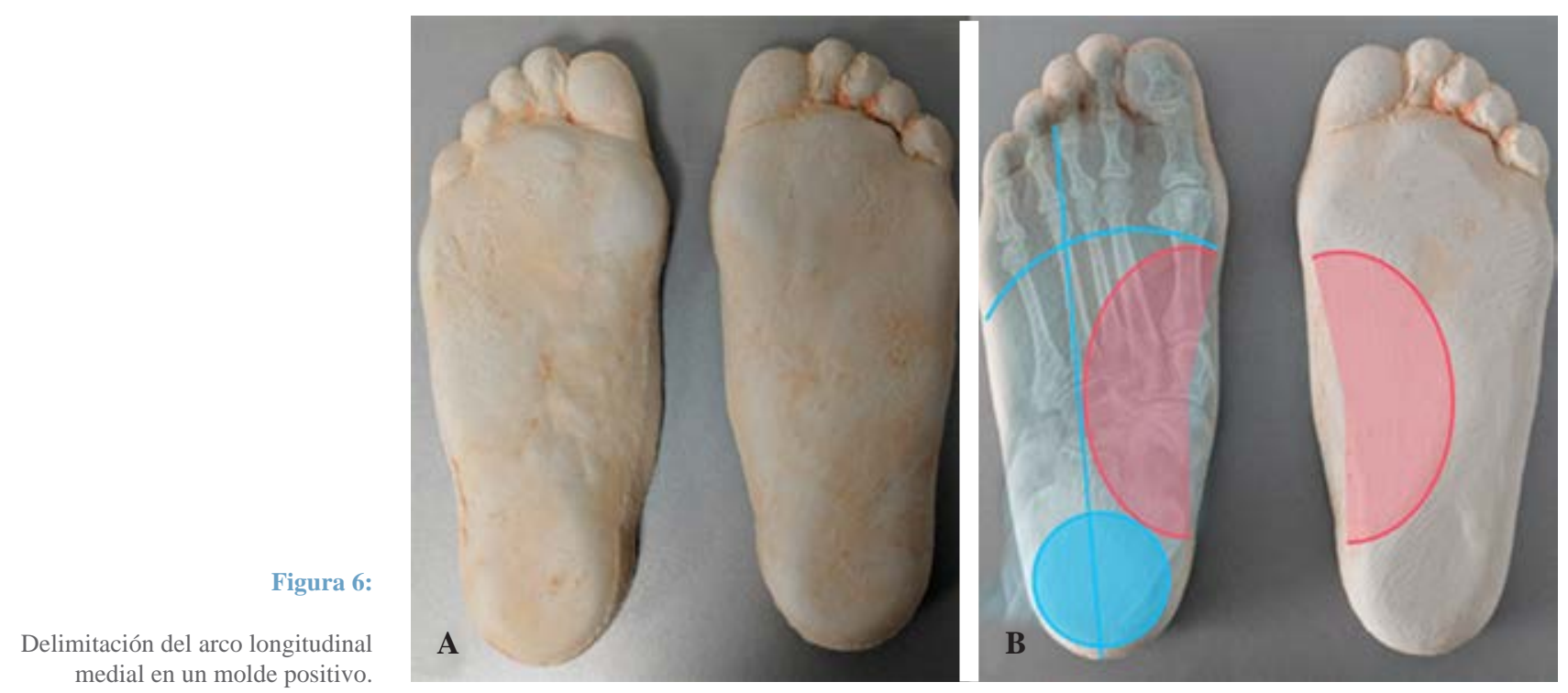

función del grado de varismo, cuyo material de elección serán EVAs de alta densidad que, calentados mediante una pistola de aire caliente, se adaptan perfectamente a la superficie plantar de la plantilla proporcionando el efecto de pronación deseado en el soporte.

\section{Resultados}

La edad de los pacientes oscilaba entre 13 y 68 años con una edad media de 48.8 a. No se encontraron diferencias en cuanto al sexo (cinco hombres y cinco mujeres), en el lado (seis casos pie izquierdo y cuatro pie derecho). El seguimiento oscilaba entre 13 meses y 92 meses, con un valor medio de 51.2 meses, 2.3 años.

El resultado con ortesis fue eficaz en siete casos, pero en tres fue necesaria la cirugía. En una ocasión se realizó únicamente una osteotomía valguizante de calcáneo (caso 10 ), en los otros dos (caso 3 y 4), que corresponden al mismo paciente, a la osteotomía de calcáneo se asoció una artrodesis del arco medial, talonavicular y talonaviculocu- neiforme respectivamente. Estos casos se correspondían con los pacientes de más edad de la serie (64 a y 68 a respectivamente).

Con relación al deporte, los casos 7 y 8, de 13 y 38 años respectivamente mejoraron completamente sus molestias, reincorporándose con total normalidad a la práctica deportiva de fútbol y tenis. El caso 7 corresponde a un varón de 13 años de edad, jugador de fútbol en la cantera de un equipo de élite. Puede verse en las radiografías como se normalizó después de un año aproximado de llevar las plantillas la forma del escafoides tanto en la imagen radiológica dorsoplantar como en el perfil, aun cuando en esta proyección persiste una imagen lineal de la fractura por insuficiencia del navicular en fase de resolución (Figura 7 A-D). El caso 9 corresponde a un varón de 38 a, monitor de tenis, volvió a dar clases, utilizando al igual que los casos anteriores las plantillas a medida. En la paciente correspondiente al caso 2, bailarina de ballet de 46 a, se redujo el dolor y de manera ocasional volvió a practicar danza.
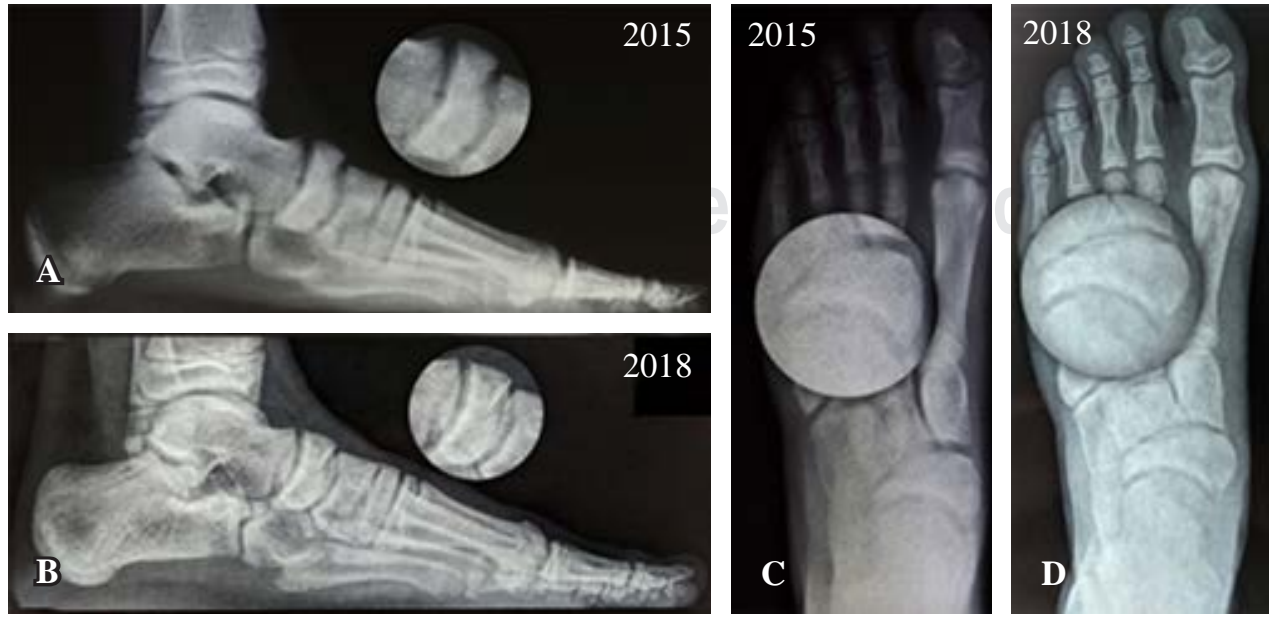

Figura 7:

A y C) RX de la primera visita. En la visión dorsoplantar se observa un adelgazamiento de la parte lateral del navicular y en el perfil una imagen compatible con una enfermedad de Müller-Weiss. B y D) Después del tratamiento durante tres años con soportes plantares el navicular recupera su forma normal en ambas proyecciones. 
En cuanto al estadio lesional, la plantilla fue eficaz en la mayor parte de los casos y de los tres casos intervenidos en dos casos (3 y 4) se trataba de una afectación bilateral en el mismo paciente en estadios IV en el pie izquierdo y $\mathrm{V}$ en el pie derecho. El caso (10) pertenecía a un grado III y fue necesaria la cirugía. También se trataba de una lesión bilateral en un mismo paciente y en el pie izquierdo, caso (9), con un estadio lesional II el tratamiento con soportes plantares fue suficiente.

\section{Discusión}

La mayoría de autores acepta que la EMW puede definirse como una displasia del navicular, que clínicamente se caracteriza por presentar con frecuencia un pie plano paradójico, ya que presenta un varo de talón. ${ }^{19}$ A partir del estadio III en muchas ocasiones se observa en la radiología, la fractura por insuficiencia del navicular con una inclinación de $45^{\circ}$ que describió Rochera $^{9}(1981)$ y aparecen de manera progresiva signos degenerativos en las articulaciones talonavicular y naviculocuneiforme donde el paciente generalmente refiere dolor.

Con los tratamientos propuestos hasta hace unos años: plantillas de inmovilización sin compensar el varo de talón y dobles artrodesis o artrodesis de las articulaciones talonavicular y naviculocuneiforme sólo se obtenían resultados mediocres. En la actualidad el tratamiento quirúrgico estándar de oro consiste en la osteotomía valguizante de calcáneo, que puede o no complementarse con una artrodesis del arco medial.

Sin embargo, en nuestra opinión, un soporte plantar a medida puede constituir una alternativa válida a la cirugía en determinados casos: estadios I-II y en algún caso de estadio III, en pacientes jóvenes de ambos sexos y en deportistas con dolor moderado.

La fabricación del soporte pasa por la captura del molde siempre en carga sin realizar compensaciones y estabilizando el positivo del molde mediante un ligera corrección del arco longitudinal medial sin hipercorregir, el objetivo en este punto es ferulizar el tarso posterior, adaptando un material rígido como el polipropileno con un grosor de 3 o $4 \mathrm{~mm}$ según la envergadura del paciente. Posteriormente añadiremos una cuña pronadora total, por lo general de unos $6 \mathrm{~mm}$ de grosor que se extenderá desde el talón hasta retrocapital de cuarto y quinto metatarsianos. Con ello obtendremos una mejora de la dinámica de la marcha del paciente así como una reducción del dolor.

Hay pocos trabajos publicados que hagan referencia al tratamiento conservador. ${ }^{10,11,12,13,14,15,16,17,18,19,20}$ Nosotros proponemos un soporte plantar con una cuña pronadora cuya altura debe estar en relación con el grado de varo que presente el retropié, junto con un soporte rígido del arco medial. Se trata de una muestra pequeña, por lo que no puede mostrarse conclusión alguna.
Podemos decir que el tratamiento conservador puede estar indicado en pacientes relativamente jóvenes y con un estadio lesional II-III. Es interesante señalar los buenos resultados obtenidos en deportistas. En enfermos con dolor importante y estadios lesionales IV-V, que por lo general se corresponde con pacientes con una edad de más de 50 años, puede también realizarse un tratamiento conservador como primera opción, pero la cirugía es más eficaz y resolutiva en la mayoría de estos casos.

Bibliografía

1. Müller W. Über eine eigenartige doppelseitige veränderung des os naviculare beim Erwachsenen (On an odd double-sided change of the tarsal navicular). Deutsche Zitschrift für Chirurgie Leipzig. 1927; 201: 84.

2. Weiss K. Über die “malazie” des os naviculare pedis (On the malacia of the tarsal navicular). Fortschritte auf dem Gebiete der Röntgenstrahlen. 1927; 45: 63-7.

3. Brailsford JF. Osteochondritis of the adult tarsal navicular. $J$ Bone Joint Surg Am. 1939; 26(1): 111-20.

4. Viladot A, Rochera R, Viladot A, Jr. Necrosis of the navicular bone. Bull Hosp Jt Dis Orthop Inst. 1987; 47: 285-93.

5. Boc SF, Feldman G. Bilateral spontaneous avascular necrosis of the navicular. Case presentation with comparative imaging. J Am Podiatr Med Assoc. 1998; 88: 41-4.

6. El-Karef E, Nairn D. The Müller-Weiss syndrome: spontaneous osteonecrosis of the tarsal navicular bone. The Foot. 1999; 9: 153-5.

7. Narváez J, Narváez JA. Osteonecrosis del escafoides tarsiano. Sem Fund Esp Reumatol. 2006; 7(3): 109-16.

8. Martinez GJ, Sebastia FE, Sierra VD, Lizaur UA. Síndrome de Müller-Weiss: necrosis espontánea del escafoides tarsiano bilateral. Revista del Pie y Tobillo. 2003; 17(2): 54-57.

9. Rochera VR, Beneyto FM, Diaz CE, Sans S Jr. Aportación al conocimiento de la escafoiditis tarsiana. Chirur Piede. 1981; 5(1): 23-32.

10. Monteagudo M, Maceira E. Management of Müller-Weiss disease. Foot Ankle Clin. 2019; 24: 89-105.

11. Maicera E, Rochera R. Müller-Weiss disease: clinical and biomechanical features. Foot Ankle Clin. 2004; 9: 105-25.

12. Maceira E. The paradoxical pes planus varus in Müller-Weiss disease. Presentado en el Second Combined Meeting of Foot and Ankle Surgeons (CoMFAS) Congress, Venecia, Italia Septiembre. 1988: 16-18.

13. Maceira E. Aspectos clínicos y biomecánicos de la enfermedad de Müller-Weiss. Rev Pie Tobillo. 1996; 10(1). 53-65.

14. Fernandez de Retana P, Maceira E, Fernandez-Valencia JA, Suso S. Arthrodesis of the talonavicular-cuneiform joints in Müller-Weiss disease. Foot Ankle Clin. 2004; 9(1): 65-72.

15. Forniciari P, Gilgen A, Zwicky I, Horn Lang T, Hintermann B. Isolated talonavicular fusion with tension band for Müller-Weiss syndrome. Foot Ankle Int. 2014; 35(12): 1316-22.

16. Lui TH. Arthroscopic triple arthrodesis in patients with Müller-Weiss disease. Foot Ankle Surg. 2009; 15(3):119-122.

17. Cao HH, Lu WZ, Tang KL. Isolated talonavicular arthrodesis and talonavicular-cuneiform arthrodesis for the Müller-Weiss disease. $J$ Orthop Surg Res. 2017; 12: 83.

18. Li SY, Myerson MS, Monteagudo M, Maceira E. Efficacy of calcaneus osteotomy for treatment of symptomatic Müller-Weiss disease. Foot Ankle Int. 2017; 38(3): 261-9.

19. Nuñez SM, Maceira SE, Fernández RP. Enfermedad de Müller-Weiss. Rev Pie Tobillo. 2007; 21(Supl. 1): 46-55.

20. Ruiz EJ, Viladot PR, Álvarez GF. Tratamiento conservador de la enfermedad de Müller-Weiss. A propósito de un caso. Rev Pie Tobillo. 2019; 33(1): 49-54. 
Protección de personas y animales: Los autores declaran que para esta investigación no se han realizado experimentos en seres humanos ni en animales.

Confidencialidad de los datos. Los autores declaran que han seguido los protocolos de su centro de trabajo sobre la publicación de datos de pacientes.
Derecho a la privacidad y consentimiento informado. Los autores declaran que en este artículo no aparecen datos de pacientes.

Financiación: Los autores declaran que este trabajo no ha sido financiado.

Conflicto de intereses. Los autores declaran no tener ningún conflicto de interés. 